\title{
Severe folate deficiency anemia associated with the use of a PARP inhibitor (olaparib) in a patient with fallopian tube cancer
}

\author{
Binoy Yohannan ${ }^{1}$, Kristi Mclntyre ${ }^{2}$, Mark Feldman*1 \\ ${ }^{1}$ Department of Internal Medicine, Texas Health Presbyterian Hospital, Dallas, Texas, United States \\ ${ }^{2}$ Department of Internal Medicine, Division of Hematology and Oncology, Texas Health Presbyterian Hospital, Dallas, Texas, \\ United States
}

Received: August 27, 2018

DOI: $10.5430 /$ crim.v6n1p1
Accepted: September 24, $2018 \quad$ Online Published: September 27, 2018

URL: https://doi.org/10.5430/crim.v6n1p1

\begin{abstract}
Treatment of cancer patients with olaparib (PARP inhibitor) is associated with an increased risk of anemia, which is seen in a majority of treated patients. However, symptomatic anemia requiring transfusion is rare. Olaparib-induced anemia can be secondary to bone marrow suppression, hemolysis or folate deficiency. We report a case of new onset severe folic acid deficiency anemia in a patient with breast and relapsed fallopian tube cancer being treated with olaparib. Complete blood count on admission showed a hemoglobin of $4.2 \mathrm{~g} / \mathrm{dl}$ and serum folate was undetectable $(<1.6 \mathrm{ng} / \mathrm{ml}$; reference range $7-31.4 \mathrm{ng} / \mathrm{ml})$. This is the second report of olaparib-induced folate deficiency anemia. She received three units packed red cell transfusion and parenteral folic acid supplementation and improved symptomatically. This case highlights the importance of recognizing folate deficiency as a reversible cause of anemia with PARP inhibitor therapy.
\end{abstract}

Key Words: Fallopian tube cancer, Olaparib, Folate deficiency anemia

\section{INTRODUCTION}

Folic acid (vitamin $\mathrm{B}_{9}$ ) is a water-soluble vitamin which has several vital functions. It is required for the repair and methylation of DNA and acts as a cofactor in numerous biological reactions. It regulates cell division and plays an important role in erythropoiesis. Folic acid deficiency is usually seen in physiological conditions such pregnancy and lactation due to increased metabolic demand for this vitamin. Other causes include chronic alcoholism, intestinal malabsorption, hemolytic anemia and exfoliative skin diseases. Certain drugs such as phenytoin and dihydrofolate reductase inhibitors such as methotrexate also may cause folate deficiency. ${ }^{[1]}$ Poly ADP ribosyl polymerase (PARP) is an enzyme that repairs DNA damage in tumor cells and in normal cells. With the goal of selectively impairing DNA repair in tumor cells, PARP inhibitors such as olaparib are being used to treat recurrent ovarian, fallopian tube and breast cancers. The PARP-1 enzyme is involved in the repair of single-stranded DNA breaks, while PARP-2 plays a pivotal role in hematopoietic homeostasis by determining the survival of stem cells and their response to oxidative stress. ${ }^{[2]}$ High levels of reactive oxygen species are observed in PARP2 deficient hematopoietic stem cells, resulting in DNA damage and because the DNA repair mechanism is ineffective,

\footnotetext{
*Correspondence: Mark Feldman, MD; Email: MarkFeldman@texashealth.org; Address: Department of Internal Medicine, Texas Health Presbyterian Hospital, Dallas, Texas, 75231, United States.
} 
it leads to activation of p53-dependent apoptotic pathways. This accelerated cellular apoptosis may be compensated for by an increase in turnover of the progenitor cells, but this replicative stress limits progenitor cell differentiation and makes the cells more susceptible to extravascular hemolysis. It has been shown that deficiency of the enzyme PARP-2 in mice results in a decrease in RBC count, enlarged erythrocyte size, the appearance of Howell-Jolly bodies and a reduced lifespan of circulating erythrocytes. ${ }^{[3]}$

Here we report a case of severe folate deficiency anemia induced by the PARP inhibitor olaparib in a patient with advanced fallopian tube cancer.

\section{Case presentation}

A 76-year-old female with advanced fallopian tube cancer with peritoneal carcinomatosis on olaparib therapy presented to the emergency department with fatigue, generalized weakness and dyspnea on exertion. She denied fever, chills, night sweats, weight loss, easy bruising or bleeding. She also denied abdominal pain, melena and hematochezia. There was no history of alcohol abuse or any dietary restriction.

She had stage III B high grade papillary serous carcinoma of the fallopian tube with omental metastasis that was diagnosed 11 years ago. At that time, peritoneal washings were negative for malignant cells. She underwent total abdominal hysterectomy and salpingoophorectomy followed by six cycles of systemic chemotherapy with taxol and carboplatin and consolidation with three cycles of intraperitoneal chemotherapy with the same drugs. One year later, she had a biopsyproven intraabdominal recurrence that was treated with carboplatin, gemcitabine and bevacizumab. This therapy was continued for 6 years but was eventually discontinued due to progression of disease and bevacizumab-induced nephrotic syndrome. She was switched to docetaxel for the next 2 years. Two years prior to the current admission, she was diagnosed with stage II B, HER2 positive breast cancer which was treated with mastectomy followed by transtuzumab followed by maintenance therapy with anastrozole. Docetaxel was discontinued. Genetic testing for a BRCA mutation was negative. The fallopian tube cancer was progressing despite multiple types of prior chemotherapy and she was started on olaparib $300 \mathrm{mg}$ twice daily 2 months prior to the current admission. Other medical problems included hypertension, bipolar disorder and gastroesophageal reflux disease. A family history included a maternal grandmother with gastric cancer at age 76, a maternal first cousin with breast cancer at age 45 , and a maternal aunt with ovarian cancer (BRCA negative).

On physical examination her temperature was $98.3^{\circ} \mathrm{F}$ with blood pressure 100/68 mmHg, pulse 99 beats per min and respiratory rate 18 per minute. She looked very pale but had no icterus or lymphadenopathy. She had moist mucous membranes and the oropharynx was clear with no evidence of cheilitis or glossitis. She had a soft ejection systolic murmur at the apex and normal vesicular breath sounds. Her abdomen was soft and non-tender without hepatosplenomegaly or ascites. Neurological examination was unremarkable without evidence of peripheral neuropathy. Digital rectal examination showed no hemorrhoids or fissures and a fecal occult blood test was negative.

Her routine blood tests on admission showed profound anemia with a hemoglobin level of $4.1 \mathrm{~g} / \mathrm{dl}$ (see Table 1).

Table 1. Laboratory test results before and after olaparib treatment

\begin{tabular}{|c|c|c|c|c|c|}
\hline & Reference range & $\begin{array}{l}6 \text { months prior } \\
\text { to admission }\end{array}$ & $\begin{array}{l}2 \text { months prior } \\
\text { to admission* }\end{array}$ & Admission & 2 week follow up \\
\hline Hemoglobin (g/dl) & $12-15$ & 10.3 & 12.1 & 4.1 & 9.1 \\
\hline Hematocrit (\%) & $34.9-44.5$ & 33 & 37 & 12.2 & 27 \\
\hline $\operatorname{MCV}(\mathrm{fl})$ & $80-96$ & 107 & 98.3 & 96 & 95 \\
\hline Reticulocyte count (\%) & $0.5 \%-1.5 \%$ & & & $0.39 \%$ & $7.42 \%$ \\
\hline WBC count $/ \mathrm{mm}^{3}$ & $4,500-11,000$ & 9,500 & 4,600 & 10,000 & 6,700 \\
\hline Platelet count $/ \mathrm{mm}^{3}$ & $150,000-400,000$ & 210,000 & 177,000 & 200,000 & 236,000 \\
\hline Serum ferritin (ng/ml) & $20-200$ & & & 215 & \\
\hline Serum iron $(\mu \mathrm{g} / \mathrm{dl})$ & $40-155$ & & & 233 & \\
\hline Serum transferrin (mg/dl) & $173-360$ & & & 182 & \\
\hline Serum vitamin $B_{12}(n g / L)$ & $>300 \mathrm{ng} / \mathrm{L}$ & 713 & & 995 & \\
\hline Serum folic acid (ng/ml) & $7-31.4 \mathrm{ng} / \mathrm{ml}$ & 10.0 & & undetectable & 54.0 \\
\hline
\end{tabular}

Note. ${ }^{*}$ Prior to beginning olaparib treatment; ${ }^{\#}$ After discontinuing olaparib and starting 3 mg folic acid daily 
The platelet and white blood cell counts were normal. Serum folate was undetectable $(<1.6 \mathrm{ng} / \mathrm{ml}$; reference range, 7 $31.4 \mathrm{ng} / \mathrm{ml}$ ) confirming severe folic acid deficiency. Serum $\mathrm{B}_{12}$, iron studies and a coagulation profile were essentially normal. Her last recorded hemoglobin level 2 months prior to starting olaparib was $12.1 \mathrm{~g} / \mathrm{dl}$.

She received 3 units of packed red cell transfusions and $3 \mathrm{mg}$ folic acid by mouth daily. The hemoglobin increased to $9.1 \mathrm{~g} / \mathrm{dl}$ and she improved symptomatically. Olaparib was discontinued and she was discharged home on $3 \mathrm{mg}$ folic acid daily with plans to resume the olaparib at reduced dose in 6 weeks.

\section{Discussion}

The hematological toxicity profile of PARP inhibitors is similar to many cytotoxic chemotherapy agents and includes anemia, neutropenia and thrombocytopenia. The majority of anemia events in patients on olaparib therapy are mild or moderate; grade $\geq 3$ events are rare. Anemia in patients treated with olaparib can be multifactorial, either from bone marrow failure, hemolysis or folate deficiency. Olaparib is a PARP-1, 2 and 3 inhibitor approved in the US for the treatment of recurrent epithelial ovarian, fallopian tube, primary peritoneal and germ line BRCA mutated breast cancer. ${ }^{[4]}$

Interestingly, studies of the long-term safety of olaparib have shown that almost all patients with anemia also had evidence of macrocytosis, even with normal serum folate, vitamin $\mathrm{B}_{12}$ and TSH levels. The mechanism of this olaparib-induced macrocytic anemia is unclear. ${ }^{[5]}$

Folate deficiency can also occur in patients treated with olaparib. Of 6 ovarian cancer patients on olaparib therapy, 4 developed severe folate deficiency within 4 weeks of starting the drug. Three patients required an olaparib dosage reduction due to transfusion dependent anemia, with a mean time to transfusion of 39 days from initiation of therapy. Almost all patients had an excellent response to oral folate replacement with appropriate improvement in hemoglobin and transfusion independence. ${ }^{[6]}$ It is as yet unclear what folate replacement therapy will do in terms of the anti-tumor drug efficacy of olaparib. Whereas folate deficiency is ordinarily associated with macrocytic anemia, it was not uncommon to have a normocytic anemia in these 4 ovarian cancer patients, as in our patient with fallopian tube cancer. Hence, the initial evaluation of normocytic anemia in a cancer patient on olaparib therapy should include serum folate levels.

The mechanism of severe folate deficiency in cancer patients treated with PARP inhibitors such as olaparib is not clearly understood. It could be secondary to high folate consumption from hemolysis and increased RBC turnover. Our patient did not appear to be actively hemolyzing since her reticulocyte count was low and the indirect serum bilirubin level was normal. Folate deficiency could also be due to intestinal malabsorption. Dietary folate is predominantly present in polyglutamyl forms which undergo hydrolysis to monoglutamyl derivates that are absorbed both in the small intestine and colon, with a decreasing absorptive gradient from jejunum to colon. Folate is then transported into intestinal epithelial cells via folate transporters which include the reduced folate carrier (RFC), the proton-coupled folate transporter (PCFT), and the folate receptor proteins, FR $\alpha$ and FR $\beta .{ }^{[7]}$ Nuclear respiratory factor 1 (NRF1) is a major inducible transcriptional regulator of $P C F T$ gene expression and is under the influence of PARP-1. It can be postulated that PARP-1 inhibition leads to inactivation of NRF1 and thereby downregulation of PCFT expression, thus compromising intestinal folate absorption. ${ }^{[8]}$

The proximal renal tubules play a crucial role in regulating body folate homeostasis by reabsorbing the filtered vitamin, thus preventing its losses in the urine. Theoretically, olaparib could reduce renal tubular folate reabsorption.

The complete blood cell count should be monitored weekly during the first month of olaparib therapy and then monthly thereafter. Also, before starting a PARP inhibitor, clinicians should ensure patients have recovered from the myelosuppression of previous cytotoxic chemotherapy. ${ }^{[9]}$ If a patient develops severe hematological toxicity associated with olaparib therapy with refractory cytopenia, bone marrow biopsy and cytogenetic analysis is recommended to rule out therapy- related myelodysplastic syndrome or acute myeloid leukemia. ${ }^{[9,10]}$

\section{Conclusion}

Severe hematologic toxicity is one of the main treatment related adverse effects of PARP inhibitors in cancer patients and therefore regular monitoring of complete blood cell counts is recommended. Folate deficiency can occur in cancer patients treated with olaparib and the average time to depletion of folate stores is 4 to 6 weeks after initiating of treatment. The exact mechanism of folate deficiency is unknown, and further studies are required to explore the potential association between PARP inhibition and the folate pathway. Also, it is critical for clinicians to be aware of folate deficiency as a reversible cause of anemia, as the folate supplements can improve the safety and tolerability of olaparib therapy.

\section{CONFLICTS OF INTEREST Disclosure}

The authors declare that they have no competing interests. 


\section{REFERENCES}

[1] Wani NA, Hamid A, Kaur J. Folate status in various pathophysiological conditions. IUBMB Life. 2008; 60(12): 834-842. PMid:18942083. https://doi.org/10.1002/iub.133

[2] Bai P. Biology of Poly ADP-Ribose Polymerases: The factotums of cell maintanance. Molecular cell: Review. 2015; 58: 947-958. PMid:26091343. https://doi.org/10.1016/j.molcel. 2015. 01.034

[3] Farrés J, lacuna L, Martin-Caballero J. PARP-2 sustains erythropoiesis in mice by limiting replicative stress in erythroid progenitors. Cell Death and Differentiation. 2015; 22: 1144-1157. PMid:25501596. https://doi.org/10.1038/cdd. 2014. 202

[4] Available from: https://www.accessdata.fda.gov/drugsat fda_docs/nda/2017/2085580rig1s000MultidisciplineR. pdf

[5] Van der Noll R, Marchetti S, Steeghs N, et al. Long-term safety and anti-tumor activity of olaparib monotherapy after combination with carboplatin and paclitaxel in patients with advanced breast, ovarian or fallopian tube cancer. British Journal of Cancer. 2015; 113: 396-402. PMid:26180927. https ://doi.org/10.1038/bjc. 2015.256
[6] Shammo JM, Usha L, Richardson KJ, et al. Olaparib-induced severe folate deficiency in women with relapsed ovarian cancer: first report and case series. J Clin Oncol. 2018; 36(suppl): e17540. https : //doi.org/10.1200/JC0.2018.36.15_suppl.e17540

[7] Visentin M, Diop-Bove N, Zhao R. The intestinal absorption of folates. Annu Rev Physiol. 2014; 76: 251-274. PMid:24512081. http s://doi.org/10.1146/annurev-physiol-020911-153251

[8] Hossain MB, Ping Ji, Ramakrishnan A, et al. Poly(ADP-ribose) Polymerase 1 Interacts with nuclear respiratory factor 1 (NRF-1) and plays a role in NRF-1 transcriptional regulation. Journal of Biological Chemistry. 2009; 284(13): 8621-32. PMid:19181665. https : //doi.org/10.1074/jbc.M807198200

[9] Friedlander M, Banerjee S, Mileshkin L. Review Article: Practical guidance on the use of olaparib capsules as maintenance therapy for women with BRCA mutations and platinum-sensitive recurrent ovarian cancer. Asia-Pacific Journal of Clinical Oncology. 2016; 12: 323331. PMid:27917619. https ://doi .org/10.1111/ajco.12636

[10] Chase DM, Patel S, Shields K. Profile of olaparib in the treatment of advanced ovarian cancer. Int J Wom Health. 2016; 8: 125-129. PMid:27186080. https ://doi .org/10.2147/IJWH. S55906c 